\title{
Perceptual Coding of Haptic Data in Time-Delayed Teleoperation
}

\author{
lason Vittorias* \\ Institute of Automatic \\ Control Engineering (LSR) \\ Technische Universität \\ München
}

\author{
Julius Kammerl ${ }^{\dagger}$ \\ Institute of Communication \\ Networks (LKN) \\ Technische Universität \\ München
}

\author{
Sandra Hirche \\ Institute of Automatic \\ Control Engineering (LSR) \\ Technische Universität \\ München
}

\author{
Eckehard Steinbach ${ }^{\S}$ \\ Institute of Communication \\ Networks (LKN) \\ Technische Universität \\ München
}

\begin{abstract}
In telepresence and teleaction systems the haptic communication channel plays a central role. As it closes a global control loop any introduced communication delay possibly destabilizes the system and impairs the performance. The scattering theory is known to solve these stability issues by transmitting wave variables instead of haptic signals, i.e. force and velocity, over the communication channel. For stability and performance additionally high packet rates are required stressing the underlying network resources. Perceptual coding techniques of haptic signals, such as the Weberinspired deadband approach, are known to successfully reduce the packet rate on the haptic channel. However, as wave variables do not directly represent haptic information but a linear transformation of both signals, perceptual coding is not directly applicable anymore. In this paper, we present a novel control scheme as well as a modification of the deadband approach to take advantage of the stabilization ability of the wave variables while allowing perceptual coding on the communication channel. Simulation results and comparison with the wave variables architecture indicate improved data compression for same degree of transparency for purely stiff as well as free environments.
\end{abstract}

Index Terms: perceptual coding, haptic data compression, wave variables, scattering transformation

\section{INTRODUCTION}

The prefix tele origins from the Greek word " $\tau \eta \lambda \varepsilon$ " meaning on remote distance. Telepresence and teleaction (TPTA) systems allow humans to experience and to operate in a remote or inaccessible environment. Applications are not limited to remote and even dangerous environments but also range from scaled operation (e.g. micro assembly) over safety critical applications all the way to minimally invasive surgery. To enable the human operator to immerse into the remote environment, multimodal sensory and command data needs to be communicated over a communication channel, see Figure 1 for a schematic overview of a TPTA system.

As in TPTA systems haptic signal are sent bidirectionally, a global control loop is closed over the communication system. Communication time delay between the human operator (OP) and the teleoperator (TO) is often unavoidable and can put the system's stability at risk [1] or at least deteriorates the transparency. Transparency refers to the performance of the TPTA system and is provided if the human user cannot distinguish between direct and teleoperated interaction with the environment. Two main sources of latency in haptic data communication are identified, namely haptic signal processing time due to encoding/compression/decompression/decoding and transmission time

\footnotetext{
*e-mail: vittorias@tum.de

†e-mail: kammerl@tum.de

†e-mail: s.hirche@ieee.org

§e-mail: eckehard.steinbach@tum.de
}

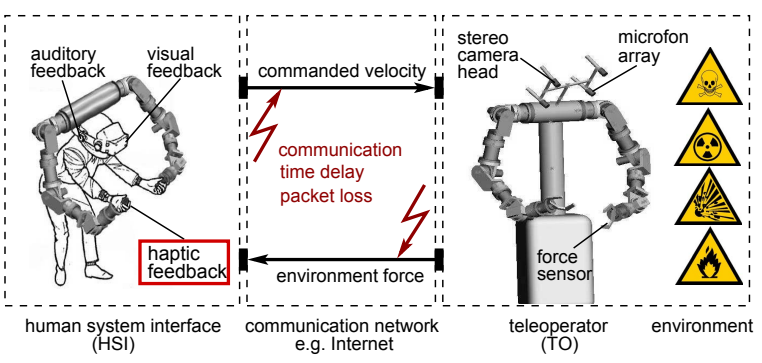

Figure 1: Multimodal telepresence and teleaction system

delay. For the sake of stability and transparency, both time delay components are desired to be as small as possible.

For the signal processing part this means that instead of blockwise sending haptic data each sample needs to be sent individually over the network. Given a packet-based communication network this results in a packet rate as high as the sampling rate of the local control loops at the human system interface (HSI) and the teleoperator. The increased packet load as well as additional data overhead due to the transmission of packet header information becomes a critical factor and corresponding network overhead can even dominate compared to the actual payload data. Our previous work has shown that these challenges can be successfully addressed by the so called deadband approach [9]. By applying a simple, yet effective perceptual model of the human haptic perception system, it allows for reducing the amount of haptic samples considered to be relevant for transmission which also results in a reduced packet rate over the communication channel.

The transmission time delay component is typically given by the communication network characteristics and is not controllable. To still enable telepresence applications in the presence of significant transmission time delay several control architectures have been developed to address the stability issues $[1,4,8,19]$. In particular, control architectures based on the scattering theory [1] are often deployed to stabilize TPTA systems in the presence of constant time delay. These approaches ensure the desired stability by preserving passivity within all elements of the communication system and the control architecture of a TPTA system. Instead of haptic signals wave variables are transmitted with the effect that arbitrary but constant time delay between the operator and the teleoperator no longer has a destabilizing effect. Wave variables represent a linear transformation of the original haptic signals velocity and force. It is unknown so far whether perceptual resolution limits like the Weber fraction can be appropriately defined in the wave variable domain or how existing ones on haptic data can be transformed. Accordingly, it s difficult to apply perceptual arguments for data compression and encoding in the wave variable domain.

In this paper we present a novel control scheme, based on the scattering theory, which allows the transmission and encoding of haptic data, while in parallel preserves stability under constant, known time-delay. As shown in [10] coding artefacts may introduce energy into the system, the applied encoding schemes must be 
carefully chosen. In line with that we also present a modification of the deadband compression approach for haptic data in this work. The effect on the transparency and the compression ratio is evaluated in simulation studies. The results are compared with an earlier proposed method by the same authors [11].

The remainder of this paper is structured as follows. In Section 2 background on time-delayed teleoperation and perceptual coding of haptic signals is discussed and previous work on this area is presented. In Section 3 we present a novel control architecture for time-delay teleoperation and the corresponding perceptionoriented haptic data reduction algorithm. The proposed approach is evaluated and compared with alternative approaches in a simulation study in Section 4.

\section{BACKGROUND}

\subsection{Time-delayed teleoperation with wave variables}

Different architectures for teleoperation under constant [1] or timevarying time delay $[5,18]$ and even packet loss [2] have been presented in the recent literature. For a survey on TPTA system control architectures with time delay refer to $[12,15]$. Some of the approaches like the impedance control scheme in [4] provide stable interaction for constant time delay but can be applied to linear time-invariant systems only. The scattering transformation in [1], later referred to as wave variable approach in [19], ensures stability of the global haptic control loop within TPTA systems under the assumption of arbitrary large, constant time delay in the communication channel. The stability arguments are based on the passivity concept which is very versatile as it may cope also with nonlinear and time-varying systems. For this reason, the wave variables architecture together with the passivity framework is chosen as method to guarantee stability in this work.

\subsubsection{Passivity}

Passivity is an energy-based concept characterizing the system by only analyzing its input/output behavior. It provides sufficient, but not necessary, input/output stability conditions.

The systems we consider are continuous time, non-linear time invariant dynamical systems described by the equations

$$
(\Sigma)\left\{\begin{aligned}
\dot{x} & =f(x, u), \quad x \in \mathbb{R}^{n} \\
y & =h(x, u), \quad u, y \in \mathbb{R}^{m}
\end{aligned}\right.
$$

where $x, u, y$ are the state input and output vectors respectively, and $f(0,0)=h(0,0)=0$. The function $f$ is locally Lipschitz, and $h$ is continuous. Thus, for each fixed initial state $x(0),(1)$ defines a causal mapping from the input signal $u(\cdot)$ to the output signal $y(\cdot)$.

Define the power $P_{\text {in }}$ entering a system as the scalar product between the input vector $u$ and the output vector $y$ of the system. Note that this power does not necessarily correspond to any actual physical power if the input/output variables are not chosen to be co-located velocity and force signals. In addition, define a lowerbounded energy storage function $S$ and a non-negative power dissipation function $P_{\text {diss }}$, which again need not represent true physical quantities. With these definitions a system is said to be passive if it obeys

$$
P_{\text {in }}=u^{T} y=\frac{d S}{d t}+P_{\text {diss }}
$$

i.e. if the power is either stored or dissipated. A further and important practical feature of the passivity formulation is its closure properties. This implies that the combination of two passive systems connected in either a feedback or parallel configuration is again passive [22].

A bilateral teleoperation system can be represented as cascaded feedback interconnection of its components, i.e. the human, the HSI, the TO and the environment, see e.g. [19]. The dynamics of the human operator and the remote environment are typically

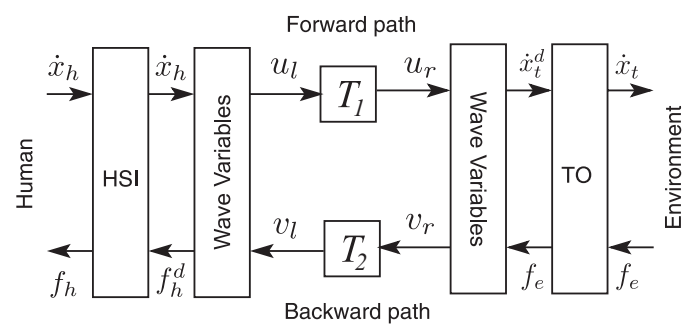

Figure 2: Standard wave variable architecture

largely unknown. Nevertheless, a trained human being can be considered to interact passively with passive environments as Hogan shows in [14]. Most environments can be considered to be passive systems as well. Additionally, the HSI and TO are usually passive or can be made passive by appropriate control. If the communication system is passive as well, then passivity of the overall interconnected system and such stability can be deduced.

In this paper the focus is on the passivity/passification of the communication channel including the haptic signal compression and time-delayed transmission. The passivity of the communication system can also be nicely analyzed using a 2-port model instead of the feedback description. Note that in the corresponding description above, all power was declared positive if it were entering the system and increasing the stored energy. In the 2-port case, however, it makes sense to distinguish a left-hand port $l$, which has positive power entering the system, and a right-hand port $r$, which has positive power exiting the system. Therefore the total power flow, which governs the stored energy change according to (2), is now given by

$$
P_{\text {in }}=u_{l} y_{l}-u_{r} y_{r}
$$

where $u_{\{l, r\}}$ and $y_{\{l, r\}}$ the input and output of the left and right hand side of the two port respectively. The cascade of such 2-port elements is still a passive 2-port element as shown in [19]. The bilateral teleoperation system can in fact alternatively be represented by a cascade of 1- and 2-ports as illustrated in Figure 2. Human and environment are represented by 1-ports, the TO, the HSI and the communication system which is composed of the wave variable transformation blocks and time-delayed transmission lines are 2-ports.

Note that the controller at TO and HSI as well as the communication channel are implemented in discrete time. It is well-known that the passivity property in a sampled data system may be lost due to sample and hold components [6]. However, in typical TPTA systems the local controller sampling rates are $1 \mathrm{kHz}$ and more; they can be considered high enough to neglect this issue. For stability analysis we will consider the subsystems human/controlled HSI and controlled TO/environment as quasi-continuous.

\subsubsection{Wave variables}

The scattering transformation and passivity theory for teleoperation systems was originally developed by Anderson and Spong [1] and later extended to the concepts of wave variables by Niemeyer and Slotine [19]. The wave variable control architecture is illustrated in Figure 2. Here, $\dot{x}_{h}$ represents the velocity signal at the human system interface, $\dot{x}_{t}^{d}$ the desired velocity at the teleoperator, $f_{e}$ is the environment interaction force, and $f_{h}^{d}$ the desired force to be displayed by the HSI. The wave variables are given by 


$$
\begin{array}{ll}
u_{l}=\frac{1}{\sqrt{2 b}}\left(f_{h}^{d}+b \dot{x}_{h}\right), & u_{r}=\frac{1}{\sqrt{2 b}}\left(f_{e}+b \dot{x}_{t}^{d}\right), \\
v_{l}=\frac{1}{\sqrt{2 b}}\left(f_{h}^{d}-b \dot{x}_{h}\right), & v_{r}=\frac{1}{\sqrt{2 b}}\left(f_{e}-b \dot{x}_{t}^{d}\right),
\end{array}
$$

where $b \geq 0$ defines the characteristic impedance associated with the wave variables and represents a tuning parameter.

The wave variables $u_{l}$ (forward path) and $v_{r}$ (backward path) are transmitted over the communications network and arrive at the corresponding receiver with a time delay $T_{1}>0$ and $T_{2}>0$ respectively so

$$
u_{r}(t)=u_{l}\left(t-T_{1}\right) \quad \text { and } \quad v_{l}(t)=v_{r}\left(t-T_{2}\right) .
$$

Assuming 2-port element theory the power inflow into the communication block including the wave variable tranformation blocks at any time is given by

$$
P_{\text {in }}(t)=\dot{x}_{h}(t) f_{h}^{d}(t)-\dot{x}_{t}^{d}(t) f_{e}(t)
$$

Rearranging (3), inserting in (4) and integrating yields

$$
\left.\int_{0}^{t} P_{i n}(\tau) d \tau=\int_{0}^{t} u_{l}^{2}(\tau)-u_{r}^{2}(\tau)+v_{r}^{2}(\tau)-v_{l}^{2}(\tau)\right) d \tau .
$$

As the extended $L_{2}$ norm of the output of a delay operator with arbitrary large constant delay is always smaller than the extended $L_{2}$ norm of its input, i.e. as the constant delay operator has a small gain property, the following holds

$$
\int_{0}^{t}\left(u_{l}^{2}(\tau)-u_{r}^{2}(\tau)\right) d \tau \geq 0 \quad \text { and } \quad \int_{0}^{t}\left(v_{r}^{2}(\tau)-v_{l}^{2}(\tau)\right) d \tau \geq 0 .
$$

Consequently, the integral in (5) is always positive, i.e. the communication 2-port is passive for arbitrary large constant delays. For more details see [19].

\subsection{Perceptual coding of haptic data based on the dead- band approach}

Surprisingly, compression of haptic data has not been studied intensively so far. To enable telepresence and teleaction across ratelimited communication channels, e.g. in space and underwater, efficient and powerful methods to communicate and process haptic data signals are of utmost importance. A perception-based data reduction technique, the deadband approach, is introduced in $[9,13]$ and enables to reduce the packet rate within a packet-switched communication network.

The deadband approach is a lossy perceptual coding approach for haptic signals that exploits human haptic perception limits using Weber's law of Just Noticeable Differences (JND). It reduces the packet rate by removing data that is considered to be imperceivable by the human. A compression ratio of up to $90 \%$ on a 2-channel force-velocity teleoperation systems is achieved without significantly impairing human immersiveness as empirically shown in human user studies $[9,13]$. In the following, its background, principle and application in haptic data compression are explained.

\subsubsection{Weber's law}

Psychophysical studies revealed that haptic perception of force, velocity, pressure, etc. approximately follow the well-known WeberFechner's law, which says that the intensity of a stimulus relates logarithmically to the induced sensation in the human brain $[3,7,23]$. The psychophysical perception of a signal change is therefore proportional to the stimulus itself [24]

$$
\frac{\Delta I}{I}=\kappa \quad \text { or } \quad \Delta I=\kappa I
$$

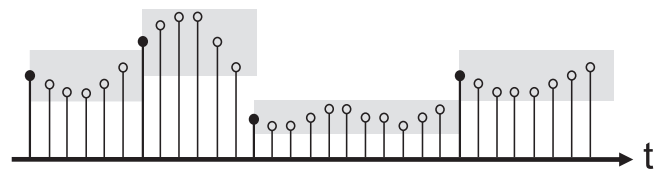

Figure 3: Principle of the Weber-inspired deadband approach

where $I$ is the initial stimulus and $\Delta I$ is the so called Difference Threshold or the Just Noticeable Difference (JND). It describes the smallest amount of change of stimulus $I$ which can be detected. The constant $\kappa$ describes the linear relationship between the JND and the initial stimulus $I$.

\subsubsection{Deadband approach}

With the deadband approach data are sent over the communication channel only if the difference between the most recently sent sample $x\left(t^{\prime}\right)$ and the current value $x(t)$ where $t>t^{\prime}$ exceeds a threshold $\Delta_{x\left(t^{\prime}\right)}$

$$
\left|x\left(t^{\prime}\right)-x(t)\right|>\Delta_{x\left(t^{\prime}\right)} .
$$

Using the insights of Weber's law this threshold is chosen to grow proportionally with the magnitude of the signal $x$

$$
\Delta_{x\left(t^{\prime}\right)}=\kappa \cdot\left|x\left(t^{\prime}\right)\right|>0
$$

where $\kappa \in(0,1)$ is a factor that influences the size of the deadband. We intentionally use the same notation for the deadband parameter as for the JND as the proposed compression scheme is inspired by Weber's law. If the haptic signal exceeds the perception threshold then a signal update event is triggered and the packet is transmitted. The current deadband is redefined based on the update sample's intensity value. The Weber-inspired deadband is proposed in [9] and has also been called relative deadband. In the following both notions, Weber-inspired and relative, will be used interchangeably.

The principle of the Weber-inspired deadband approach is illustrated in Figure 3. Filled circles represent the discrete signal output of the deadband operator; empty circles do not violate the threshold and are thus discarded. Note that the deadband increases with the signal amplitude. By this means, we modify the haptic signal in a lossy but to the human user imperceptible manner. As only the samples which violate the deadband are considered to contain perceivable information, the proposed lossy coding scheme allows for signal-adaptive downsampling and therefore dramatically reduces the amount of samples within the haptic data streams. For example, during phases of constant velocity or force no sample is sent over the respective communication channel. At the receiver side a decoder upsamples the signal to the local HSI and TO control sampling frequency by using an extrapolation algorithm.

\section{LOCAL COMPUTATION OF WAVE VARIABLES}

In the following we present a modification of the wave variable control architecture which allows for directly sending perceptually encoded haptic signals over the communication channel while ensuring wave variable based teleoperation for TPTA systems with known constant communication latency. Expanding (3), the desired force at the HSI can alternatively be written as

$$
f_{h}^{d}(t)=2 f_{e}\left(t-T_{2}\right)+\underbrace{\left.b \dot{x}_{h}(t)-b \dot{x}_{h}\left(t-T_{1}-T_{2}\right)-f_{h}\left(t-T_{1}-T_{2}\right)\right)}_{\text {all variables locally known at the OP }},
$$

i.e. it depends on the HSI velocity and the delayed HSI velocity/force which are all locally accessible assuming some storage element that stores these signals for the round-trip time delay, and 


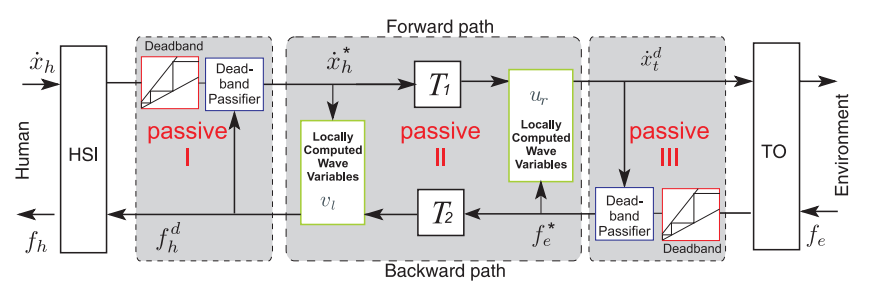

Figure 4: Proposed control architecture with coding 2-ports block I and II, and transmission 2-port block III

the environment force which is transmitted over the backward timedelayed communcation channel. Similarly, it holds for the desired velocity of the teleoperator

$$
x_{t}^{d}(t)=2 \dot{x}_{h}\left(t-T_{1}\right)+\underbrace{x_{t}\left(t-T_{1}-T_{2}\right)-f_{e}\left(t-T_{1}-T_{2}\right)-\frac{1}{b} f_{e}(t)}_{\text {all variables locally known at the TO }},
$$

where now the HSI velocity is communicated via the forward communication channel.

The transmission block of the presented approach with locally computed wave variables (LCWV) has an equivalent input/output behavior as the standard wave variable architecture, hence the passivity property is preserved and such stability is guaranteed. Observe that now haptic signals instead of wave variables are transmitted over the communication channel. This enables the use of perceptual coding schemes. In order to perform the computation, however, the local signals partially have to be stored for the roundtrip time delay $T_{1}+T_{2}: \dot{x_{h}}, f_{h}$ at the OP side and $\dot{x_{t}}, f_{e}$ at the TO side. Hence, the round-trip time delay needs to be known, which poses a limitation compared to the original wave variable approach where the exact knowledge is not required. Recent telepresence experiments between Germany and Japan over the Internet, however, showed that the time delay even over this long-distance interconnection can be considered constant [20]. Additionally, the roundtrip time delay is rather simple to measure using time-stamps.

\subsection{Modified deadband approach}

The applied coding scheme must avoid inserting additional energy into the communication system as the pure delay operator together with the wave variable approach is just lossless, i.e. any inserted energy would render the communication block including coding nonpassive. Figure 4 illustrates the our proposed control architecture as a cascade of 2-ports. Block II represents the local computation of the wave variables and the transmission of haptics signals as given by (8) and (9). As the input/output behavior of block II is equivalent to the wave variable approach, it is passive. Passivity of the communication subsystem is guaranteed if in addition to the transmission 2-port block II also the haptic coding 2-ports blocks I and III are passive. The overall system can then be represented as a cascade connection of passive 2-port elements, stability can be deduced. In the following, we present a modified deadband approach to address the passivity constraints in the haptic coding scheme.

In the deadband approach a signal-adaptive sampling is performed. A reconstruction strategy is necessary to reconstruct the (quasi-)continuous signal at the corresponding receiver side. The most commonly used zero-order hold, however, is non-passive as shown in $[6,10]$. Therefore we propose to slightly modify the transmitted values already at the sender side according to the following scheme

$$
\dot{x}_{h}^{*}(t)=\dot{x}_{h}\left(t^{\prime}\right)-\operatorname{sign}\left(f_{h}^{d}(t)\right) \Delta_{\dot{x}_{h}},
$$

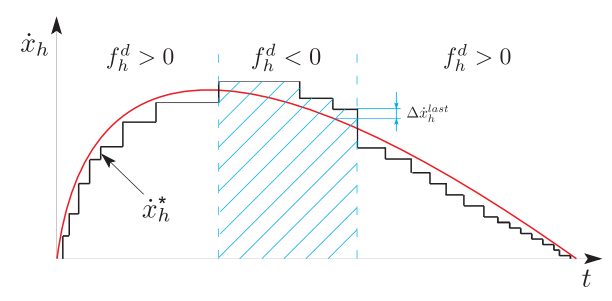

Figure 5: Effect of modified deadband approach on signal, here for OP side

at the OP side and

$$
f_{e}^{*}(t)=f_{e}\left(t^{\prime}\right)-\operatorname{sign}\left(\dot{x}_{t}^{d}(t)\right) \Delta_{f_{e}} .
$$

at the TO side where $\dot{x}_{h}^{*}$ denotes the modified velocity at the OP side and $f_{e}^{*}$ is the modified environment force value. $\Delta_{\dot{x}_{h}}$ and $\Delta_{f_{e}}$ denote the currently applied deadbands and $t^{\prime}<t$ the time instant of the most recent signal update. Depending on the sign of the desired HSI force the value is modified to upper or lower end of the deadband interval in order to dissipate energy. This is illustrated in Figure 5 for the OP side. By keeping the value within the deadband the change in signal is still considered as just not perceivable.

The proposed algorithm, denoted as deadband passifier in Figure 4, passifies the OP and TO coding 2-port subsystems, block I and III in Figure 4, and can be interpreted as a passified zero-order hold. In order to show this consider the passivity condition

$$
P_{\text {in }}=\dot{x}_{h} f_{h}^{d}-\dot{x}_{h}^{*} f_{h}^{d}=\left(\dot{x}_{h}-\dot{x}_{h}^{*}\right) f_{h}^{d}>0
$$

for the OP side and

$$
P_{\text {in }}=\dot{x}_{t}^{d} f_{e}-\dot{x}_{t}^{d} f_{e}^{*}=\dot{x}_{t}^{d}\left(f_{e}-f_{e}^{*}\right)>0
$$

for the TO side where the time argument is dropped for convenient notation. For passivity at the OP side we have to require that

$$
\operatorname{sign}\left(\dot{x}_{h}-\dot{x}_{h}^{*}\right)=\operatorname{sign}\left(f_{h}^{d}\right)
$$

and accordingly at the TO side

$$
\operatorname{sign}\left(f_{e}-f_{e}^{*}\right)=\operatorname{sign}\left(\dot{x}_{t}^{d}\right) .
$$

With the proposed algorithm this is clearly the case as can be seen from straightforward re-arranging the terms in (10) to

$$
\left(\dot{x}_{h}-\dot{x}_{h}^{*}\right)=\operatorname{sign}\left(f_{h}^{d}\right) \Delta_{\dot{x}_{h}},
$$

where $\Delta_{\dot{x}_{h}}>0$, see (7). Analogously, it can be shown for the TO side coding block. Consequently, the individual 2-port subsystems for coding and transmission are passive. Hence, the overall 2 -port communication subsystem consisting of these three cascaded passive 2-ports is passive as well. The cascade connection of those 2port systems, each of them dissipating energy, with the passive human/HSI and passive TO/environments leads then to a stable overall system.

\section{Evaluation}

To evaluate the proposed LCWV control scheme and the modified deadband scheme within a TPTA system, the packet rate reduction ability and system's transparency is investigated. Note that a haptic TPTA system is called transparent if the human operator cannot distinguish between direct interaction and tele-interaction. Ideally he or she feels directly interacting with the remote environment [21].

Our performance evaluation of the proposed control and coding architecture distinguishes two cases. We analyze the packet 

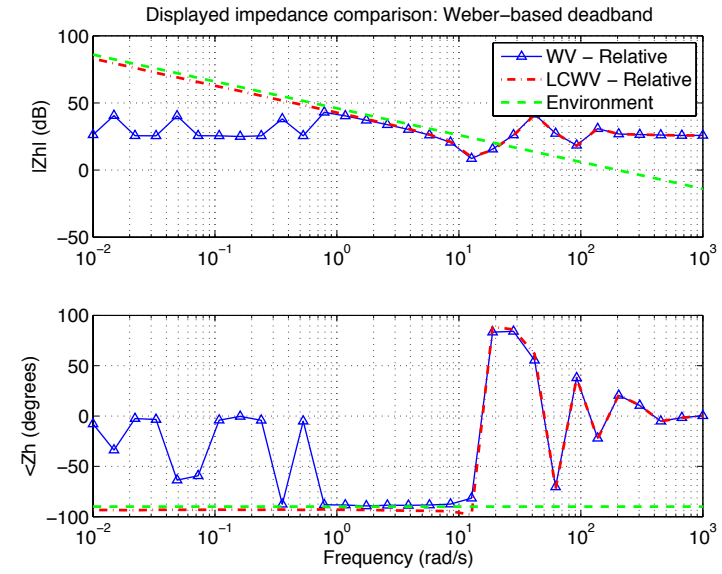

Figure 6: Bode plot of displayed impedance for spring environment: LCWV with Weber-inspired deadband with $\kappa=10 \%$; WV with Weberinspired deadband with $\kappa=10 \%$

rate when both systems show a similar degree of transparency and also compare the transparency when both systems indicate similar packet rates. In lines with the transparency criterion from [17] we analyze the displayed mechanical impedance $Z_{h}$ in comparison to the environment impedance $Z_{e}$. The mechanical impedance is given as the mapping between velocity $V$ and force $F$, if a valid linear approximation exist it can be represented in the Laplace domain as $Z(s)=\frac{F(s)}{V(s)}$. If the displayed impedance $Z_{h}$ at the OP side is equal to the environment impedance $Z_{e}$ at the TO side $Z_{e}=Z_{h}$ then the TPTA system is transparent [17]. In order to make the transparency of two approaches comparable we introduce the degree of similarity of two impedances. Particularly, we consider two impedances $Z_{h_{1,2}}$ as similar if

$$
J\left(Z_{h_{1}}, Z_{h_{2}}\right)=\int_{\omega_{\min }}^{\omega_{\max }} \frac{1}{\left|Z_{h_{1}}\right|}\left|Z_{h_{1}}(j \omega)-Z_{h_{2}}(j \omega)\right| d \omega<\varepsilon
$$

with $\varepsilon>0$ some threshold value. The deadband algorithm changes the dynamics of the system and no analytical representation or linear model for the displayed impedance $Z_{h}(s)$ can be derived. We assume, however, that a linearization of the displayed impedance around some working point exist and use identification methods to estimate its frequency response.

For the simulations we consider a haptic TPTA system where the dynamics of HSI and TO are assumed to be negligible, only the environment and the coding/transmission blocks are considered. The system is excited with sinusoidal velocity inputs within a frequency window $\left[\omega_{\min }, \omega_{\max }\right]=\left[\begin{array}{ll}10^{-2} & 10^{3}\end{array}\right] \mathrm{rad} / \mathrm{s}$ and unity amplitude. The gain and phase relation between the velocity signal and the resulting force feedback, i.e. the frequency response of the displayed impedance, is computed using a standard cross-correlation method [16]. Due to space constraint we present in this paper the results for two prototypical cases: contact with a spring environment with a spring constant of $200 \mathrm{~N} / \mathrm{m}$ and free space motion. The simulated time delay is $T_{1}=T_{2}=30 \mathrm{~ms}$ which is considered to be a realistic assumption for teleoperation scenarios on earth using modern packet-switched networks. The sampling rate of the local control loops at HSI and teleoperator is $1 \mathrm{kHz}$. Accordingly, 1000pks/s represents the standard packet rate without any data compression.

\subsection{Results}

Initially, the LCWV approach with modified deadband is applied with threshold parameter of $\kappa=10 \%$ which is an empirically
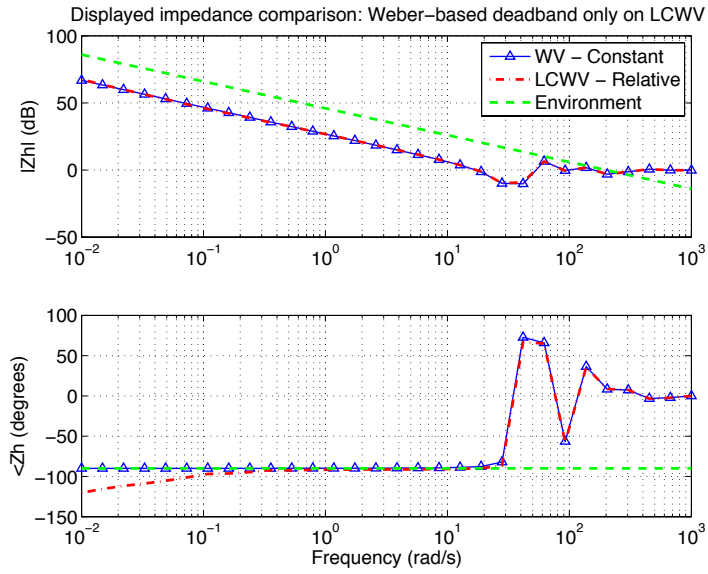

Figure 7: Bode plot of displayed impedance for spring environment: LCWV with Weber-inspired deadband with $\kappa=10 \%$; WV with constant deadband of $0.02 \sqrt{\text { Watt }}$

preferable value for transparent data reduction of haptic signals within TPTA systems, as presented in [9]. Additionally, the same Weber-inspired deadband scheme is applied on wave variables as proposed in [11], also configured with $\kappa=10 \%$. The mean packet rate measured for both methods during the simulation are:

WV - Weber-inspired deadband: $\uparrow 262 \mathrm{pks} / \mathrm{s}, \downarrow 262 \mathrm{pks} / \mathrm{s}$ LCWV - Weber-inspired deadband: $\uparrow 265 \mathrm{pks} / \mathrm{s}, \downarrow 245 \mathrm{pks} / \mathrm{s}$

where the average of the packet rate is taken over time and over all exciting frequencies, $\uparrow$ stands for the forward and $\downarrow$ for the backward channel. Both approaches show approximately the same packet rates which are substantially reduced compared to the original $1000 \mathrm{pks} / \mathrm{s}$. However, the proposed LCWV based coding scheme achieves greater transparency according to displayed stiffness indicated by the amplitude and phase impedance characteristics, i.e. $J\left(Z_{e}, Z_{h_{L C W V}}\right)<J\left(Z_{e}, Z_{h_{W V}}\right)$, as shown in Figure 6 . It can be observed that the spring characteristics is lost for the WV architecture as no integrator behavior is apparent in its frequency response. This observation complies with the results of [11] where a relative (Weber-inspired) deadband is applied in the wave variable domain directly. Interestingly, there a constant, magnitude independent deadband turns out to provide better results with respect to transparency/compression ratio than a relative deadband. Therefore, in the following we will compare our approach with the constant deadband approach for wave variables in [11].

A similar degree of transparency for the LCWV approach with $\kappa=10 \%$ and the wave variable (WV) approach with constant deadband, i.e. similar displayed impedances $J\left(Z_{h_{L C W V}}, Z_{h_{W V}}\right)=0.0015$, is achieved if the constant deadband value is chosen to be $0.02 \sqrt{\text { Watt }}$ as identified in a line search. The corresponding Bode plots are shown in Figure 7. It should be noted here that part of the deviation of the displayed impedance in both approaches from the environment impedance is also a result of the original wave variable approach even without data compression as consequence of dynamics of the bilateral controller and the time delay. This deviation usually results in stiff environment being displayed softer. The mean packet rates are strongly - by further $38 \%$ - reduced within the LCWV approach:

WV - constant deadband: $\uparrow 544 \mathrm{pks} / \mathrm{s}, \downarrow 525 \mathrm{pks} / \mathrm{s}$ LCWV - Weber-inspired deadband: $\uparrow 324 \mathrm{pks} / \mathrm{s}, \downarrow 332 \mathrm{pks} / \mathrm{s}$

Finally, free space motion is simulated using the same deadband configuration as in the previous simulation, see Figure 8 for the 

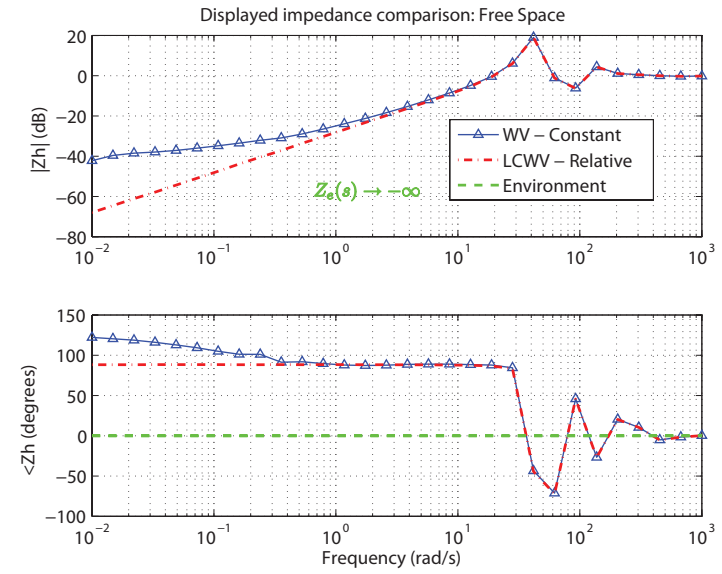

Figure 8: Bode plot of displayed impedance for free space motion: LCWV with Weber-inspired deadband with $\kappa=10 \%$; WV with constant deadband of $0.02 \sqrt{\text { Watt }}$

corresponding impedance plots. For both architectures a deviation from the ideal displayed impedance, $Z_{h}(s) \rightarrow-\infty$, is observed as the bilateral control dynamics and the time delay degrade the degree of transparency. The mean packet rate for the LCWV architecture is reduced by further $61 \%$ compared to the WV architecture, overall only $13 \%$ of the original data are transmitted:

WV - constant deadband: $\uparrow 352 \mathrm{pks} / \mathrm{s}, \downarrow 334 \mathrm{pks} / \mathrm{s}$ LCWV - Weber-inspired deadband: $\quad \uparrow 265 \mathrm{pks} / \mathrm{s}, \downarrow \mathbf{0} \mathrm{pks} / \mathrm{s}$

Qualitatively similar results are obtained for different deadband thresholds $\kappa$, i.e. different compression rates, and different environments.

\section{Conclusions}

In this paper we propose a novel approach for perceptual coding of haptic data in time-delayed teleoperation systems that improves the compression ratio compared to earlier approaches. Therefore we introduce a modification of the well-known wave variable approach which allows to transmit the non-transformed haptic signals directly and perceptually encode data on the communication channel in time-delayed teleoperation without loosing the stabilization property. In order to guarantee stability additionally a haptic data compression algorithm with a passified deadband approach is proposed. In contrast to the standard wave variable approach, the round-trip communication time delay needs to be known. The evaluation in simulation studies in spring environment and free space motions indicate that the proposed LCWV control architecture with the corresponding perceptual coding achieves higher transparency/packet rate ratios compared to the wave variable architecture.

Future work includes psychophysical experiments with human subjects for different TPTA scenarios.

\section{ACKNOWLEDGEMENTS}

This work is supported by the German Research Foundation (DFG) within the Collaborative Research Center SFB453 on "HighFidelity Telepresence and Teleaction". The authors gratefully acknowledge the contribution of Tilemachos Matiakis.

\section{REFERENCES}

[1] R. J. Anderson and M. W. Spong. Bilateral control of teleoperators with time delay. IEEE Transactions on Automatic Control, 34(5):494501, 1989.
[2] P. Berestesky, N. Chopra, and M. Spong. Theory and experiments in bilateral teleoperation over the internet. Proc. of the IEEE International Conference on Control Applications, pages 456-463, 2004.

[3] G. Burdea. Force and Touch Feedback for Virtual Reality. Wiley, 1996.

[4] H. Cho and J. Park. Impedance control with variable damping for bilateral teleoperation under time delay. JSME International Journal Series C Mechanical Systems, Machine Elements and Manufacturing, 48(4):695-703, 2005.

[5] N. Chopra, M. Spong, S. Hirche, and M. Buss. Bilateral teleoperation over the internet: the time varying delay problem. Proc. of the American Control Conference, pages 155-160, 2003.

[6] E. Colgate and G. Schenkel. Passivity of a class of sampled-data systems: Application to haptic interfaces. Journal of Robotic Systems, 14:37-47, 1997.

[7] G. Gescheider. Psychophysics. Lawrence Erlbaum, 1985.

[8] K. Hashtrudi-Zaad and S. Salcudean. Analysis of control architectures for teleoperation systems with impedance/admittance master and slave manipulators. International Journal of Robotics Research, 20(6):419445, 2001.

[9] P. Hinterseer, S. Hirche, S. Chaudhuri, E. Steinbach, and M. Buss. Perception-based data reduction and transmission of haptic data in telepresence and teleaction systems. IEEE Transactions on Signal Processing, 56(2):588-597, 2008.

[10] S. Hirche and M. Buss. Packet Loss Effects in Passive Telepresence Systems. In Proceedings of the 43rd IEEE Conference on Decision and Control, pages 4010-4015, 2004.

[11] S. Hirche and M. Buss. Transparent data reduction in networked telepresence and teleaction systems. part ii: Time-delayed communication. Presence: Teleoperators \& Virtual Environments, 16(5):532$542,2007$.

[12] S. Hirche, M. Ferre, J. Barrio, C. Melchiorri, and M. Buss. Bilateral Control Architectures for Telerobotics, pages 163-176. STAR. Springer-Verlag, Berlin, 2007.

[13] S. Hirche, P. Hinterseer, E. Steinbach, and M. Buss. Transparent data reduction in networked telepresence and teleaction systems. part i: Communication without time delay. Presence: Teleoperators \& Virtual Environments, 16(5):523-531, 2007.

[14] N. Hogan. Controlling impedance at the man/machine interface. Proc. of the IEEE International Conference on Robotics and Automation, pages 1626-1631, 1989.

[15] P. Hokayem and M. Spong. Bilateral teleoperation: An historical survey. Automatica, 42(12):2035-2057, 2006.

[16] R. Isermann. Identifikation Dynamischer Systeme I. Springer, 2 edition, 1992.

[17] D. Lawrence. Stability and transparency in bilateral teleoperation. Proc. of the 31st IEEE Conference on Decision and Control, pages 2649-2655, 1992.

[18] R. Lozano, N. Chopra, and M. Spong. Passivation of Force Reflecting Bilateral Teleoperators with Time Varying Delay. In Proc. of the 8. Mechatronics Forum, pages 954-962, Enschede, Netherlands, 2002.

[19] G. Niemeyer and J.-J. Slotine. Stable adaptive teleoperation. IEEE Journal of Oceanic Engineering, 16(1):152-162, 1991.

[20] A. Peer, S. Hirche, C. Weber, I. Krause, M. Buss, S. Miossec, P. Evrard, O. Stasse, E. S. N., A. Kheddar, and K. Yokoi. Intercontinental cooperative telemanipulation between germany and japan. IEEE/RSJ International Conference on Intelligent Robots and Systems, pages 2715-2716, 2008.

[21] G. Raju, G. Verghese, and T. Sheridan. Design issues in 2-port network models of bilateral remote manipulation. Proc. of the IEEE International Conference on Robotics and Automation, pages 13161321, 1989.

[22] R. Sepulchre, M. Jankovic, and P. Kokotovic. Constructive Nonlinear Control. Springer-Verlag, 1 edition, 1997.

[23] K. Stanney. Handbook of Virtual Environments. Lawrence Erlbaum Associates, 2002.

[24] E. Weber. Die Lehre vom Tastsinn und Gemeingefuehl, auf Versuche gegruendet. Vieweg: Braunschweig, Germany, 1851. 